\title{
Aplicaciones de la nanotecnología en el campo de la oftalmología: ¿dónde estamos?
}

\section{Nanotechnology applications in ophthalmology: An update}

\author{
Marcela Gómez-Garzón ${ }^{1}$, M. Alejandra Martínez-Ceballos ${ }^{1 *}$, Arley Gómez-López ${ }^{1,2}$ y \\ Adriana Rojas-Villarraga ${ }^{1,2}$ \\ ${ }^{1}$ División de investigaciones; ${ }^{2}$ Instituto de investigaciones. Fundación universitaria de ciencias de la salud (FUCS), Bogotá, Colombia
}

\begin{abstract}
Resumen
La nanotecnología es una disciplina que se centra en el estudio, procesamiento y aplicación de diversos materiales, aparatos y sistemas funcionales, y controla la materia a nanoescala. La nanomedicina hace referencia a sus aplicaciones para el diagnóstico, tratamiento, prevención y seguimiento de diferentes enfermedades. Los medicamentos que se administran a través de gotas para los ojos deben viajar una gran distancia y evitar varias barreras oculares para llegar al segmento posterior del ojo, lo que lleva a niveles mínimos de medicamento. Esta revisión se centra en los sistemas terapéuticos para enfermedades oculares basados en nanotecnología, y hace énfasis en las barreras que afectan la administración del fármaco ocular y en las enfermedades oculares y los nanosistemas utilizados para su manejo. Se resume la perspectiva de la nanotecnología y los desafíos existentes en la terapia y el diagnóstico de las enfermedades oculares con miras a proporcionar información y nuevas ideas para implementar tratamientos y desarrollar sistemas de manejo de enfermedades oculares intratables.
\end{abstract}

Palabras clave: Oftalmología. Nanotecnología. Nanomedicina. Nanopartículas.

\begin{abstract}
Nanotechnology is a discipline that focuses on the study, processing, and application of several materials, devices, and functional systems, controlling matter at the nanoscale. Nanomedicine refers to its applications for the diagnosis, treatment, prevention and monitoring of different diseases. Topical drug dosage forms for eye conditions must travel a great distance and overcome several eye barriers to reach the posterior segment of the eye, leading to minimum levels of medication. This review focuses on the therapeutic systems for eye diseases based on nanotechnology, emphasizing the barriers that affect the administration of ocular drugs and eye diseases and nanosystems used for their management. The perspective of nanotechnology and the challenges in the treatment and diagnosis of eye diseases are summarized, to provide information and new ideas for implementing treatments and developing management systems for intractable eye diseases.
\end{abstract}

Key words: Ophthalmology. Nanotechnology. Nanomedicine. Nanoparticles.

Correspondencia:

*María Alejandra Martínez-Ceballos

FUCS, Cl. 10 \#18-75

Fecha de recepción: 01-03-2020

Fecha de aceptación: 05-05-2020

Bogotá, Colombia

E-mail: mariamace9@gmail.com

DOI: 10.24875/RMO.M20000125

0187-4519/๑ 2020 Sociedad Mexicana de Oftalmología. Publicado por Permanyer. Este es un artículo open access bajo la licencia CC BY-NC-ND (http://creativecommons.org/licenses/by-nc-nd/4.0/).
Disponible en internet: 01-09-2020 Rev Mex Oftalmol. 2020;94(5):221-233

www.rmo.com.mx 


\section{Introducción}

La Organización Mundial de la Salud estimó que, en 2018, cerca de 1,300 millones de personas presentaban alguna forma de deficiencia visual, y las principales causas eran los errores de refracción no corregidos y la catarata. Aproximadamente existen 36 millones de ciegos debido a catarata, tracoma, cicatrices corneales, glaucoma, retinopatía diabética, degeneración macular relacionada con la edad y anomalías congénitas. Se estima que el $80 \%$ de estos casos podrían haberse evitado ${ }^{1}$.

El ojo se divide en segmento anterior y segmento posterior. El segmento anterior incluye la córnea, la conjuntiva, la cámara anterior, el iris, el cuerpo ciliar y el cristalino. La instilación de colirios oftálmicos se usa comúnmente para el tratamiento de enfermedades del segmento anterior por su fácil accesibilidad; sin embargo, las gotas oculares tópicas tienen una biodisponibilidad ocular deficiente debido a la barrera corneal, a la rápida filtración de la lágrima. El segmento posterior está compuesto por la coroides, el cuerpo vítreo y la retina. Los colirios oftálmicos deben viajar una gran distancia y atravesar varias barreras oculares para llegar al polo posterior del ojo, lo que conlleva a una baja biodisponibilidad del medicamento al llegar a su sitio de acción².

La nanotecnología es una disciplina que se centra en el estudio, el diseño, la síntesis, la manipulación y la aplicación de diversos materiales, aparatos y sistemas funcionales, y controla la materia a nanoescala (1 a 100 nanómetros). De acuerdo con la National Nanotechnology Initiative, «la esencia de la nanotecnología es la capacidad de trabajar a un nivel molecular, átomo por átomo, para crear una gran estructura, con una organización molecular fundamentalmente nueva. El objetivo es explotar estas propiedades mediante el control de estructuras y dispositivos a niveles atómicos, moleculares y supramoleculares, y aprender a fabricar y usar estos dispositivos de manera eficiente»3. Permite aplicaciones para el diagnóstico, tratamiento, prevención y seguimiento de diferentes enfermedades.

Las aplicaciones de la nanotecnología son amplias, ejemplo de ello ocurre en el campo de la biología molecular para desarrollar métodos de biodetección de secuenciación de ADN a través de secuenciadores de nanoporos ${ }^{4,5}$. En farmacología clínica, para la creación de nanomedicinas ${ }^{6}$; recientemente, la Food and Drug Administration ha aprobado algunas nanomedicinas, entre las que se mencionan según el tipo de material, nanopartículas poliméricas, categorizadas como conjugados de polímero-fármaco y arquitectura de polímeros degradables, cuyas funciones son favorecer la difusión del medicamento a través de barreras anatómicas, aumentar la biodisponibilidad y vida media del fármaco, así como favorecer mecanismos de liberación controlada. También tiene aplicación en la optimización de imágenes diagnósticas, utilizando nanopartículas inorgánicas de óxido de hierro como reactivo para mejorar el contraste de las imágenes ${ }^{7}$, entre otras.

La aplicación de tratamientos basados en la nanotecnología en enfermedades oftalmológicas es la esperanza para millones de pacientes que padecen patologías oculares. Es el caso de los nanotransportadores y las nanosuspensiones. Estos tienen la capacidad de liberar medicamentos en sitios específicos, lo cual resulta en el uso de una menor dosis de medicamento, lo que minimiza el riesgo de efectos secundarios $^{8}$. La brimonidina, ciclosporina, corticoides, implantes intravítreos de liberación sostenida, etc. son algunos ejemplos. A nivel de diagnóstico y seguimiento, la medición no invasiva de la presión intraocular (PIO), utilizada para detectar los niveles elevados de PIO, y la monitorización remota por medio de dispositivos a escala nano serán de gran beneficio para el diagnóstico precoz de la atrofia progresiva del nervio óptico y la vigilancia clínica de los pacientes con neuropatía óptica glaucomatosa ${ }^{9,10}$.

En esta revisión nos hemos centrado en los sistemas terapéuticos para enfermedades oculares basados en nanotecnología. Primero se hace una breve descripción de la anatomía ocular y las barreras que intervienen en la administración del fármaco; posteriormente se revisan las enfermedades oculares y los nanosistemas utilizados para su manejo; y finalmente se resume la perspectiva de la nanotecnología y los desafíos existentes en la terapia y el diagnóstico de las enfermedades oculares. Esta revisión proporcionará información y nuevas ideas para implementar tratamientos y desarrollar sistemas de manejo de enfermedades oculares frecuentes.

\section{Anatomía del ojo y barreras oculares}

En la tabla 1 se presentan las diferentes estructuras anatómicas oculares (Fig. 1), y se destaca su grosor, funciones y fisiología, así como su constitución. Estas características especiales pueden facilitar o impedir la acción de medicamentos tópicos oculares. Así mismo, se da un panorama general de las posibles dianas de acción, lo que permite comprender la implementación de los avances de la nanotecnología en oftalmología. 


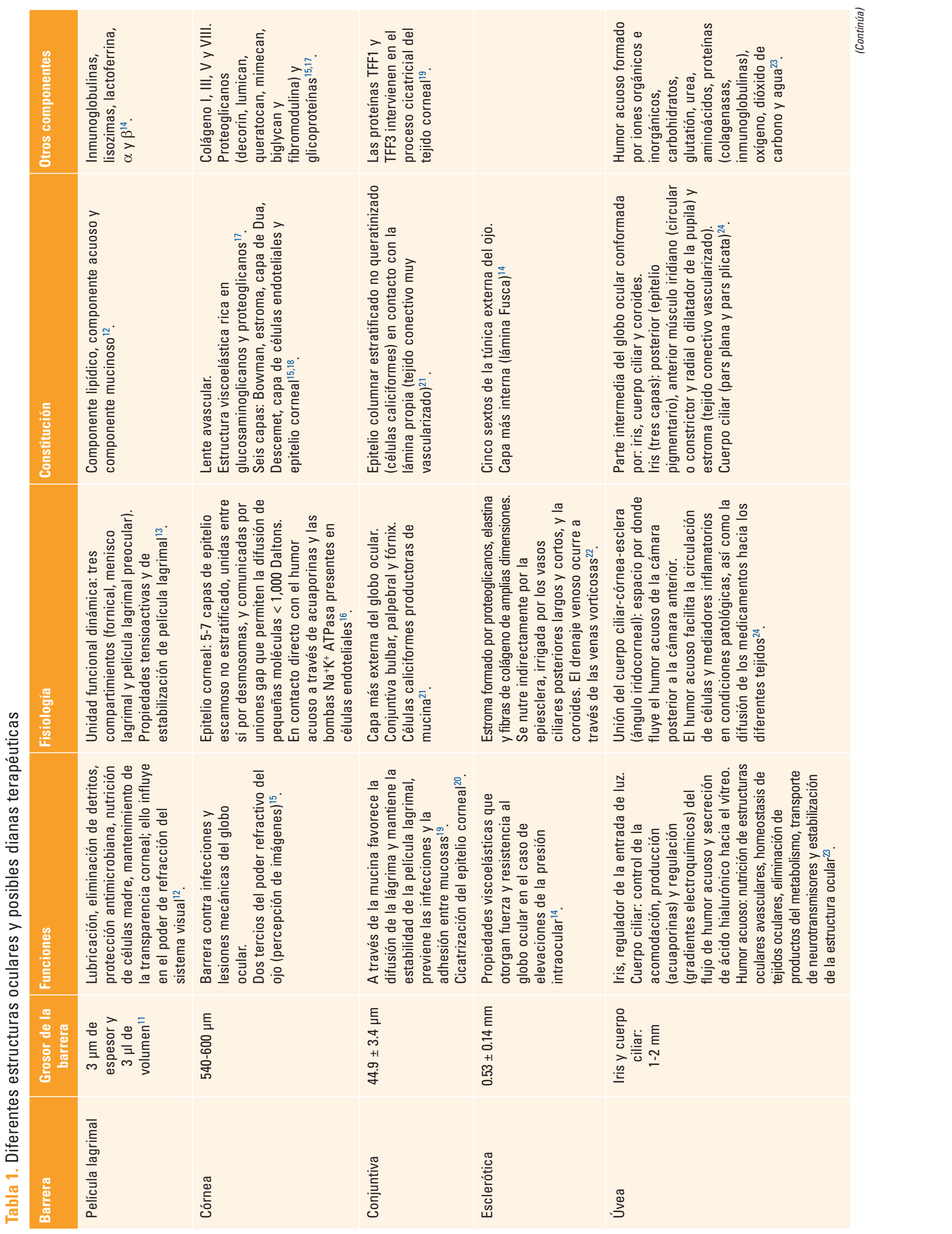




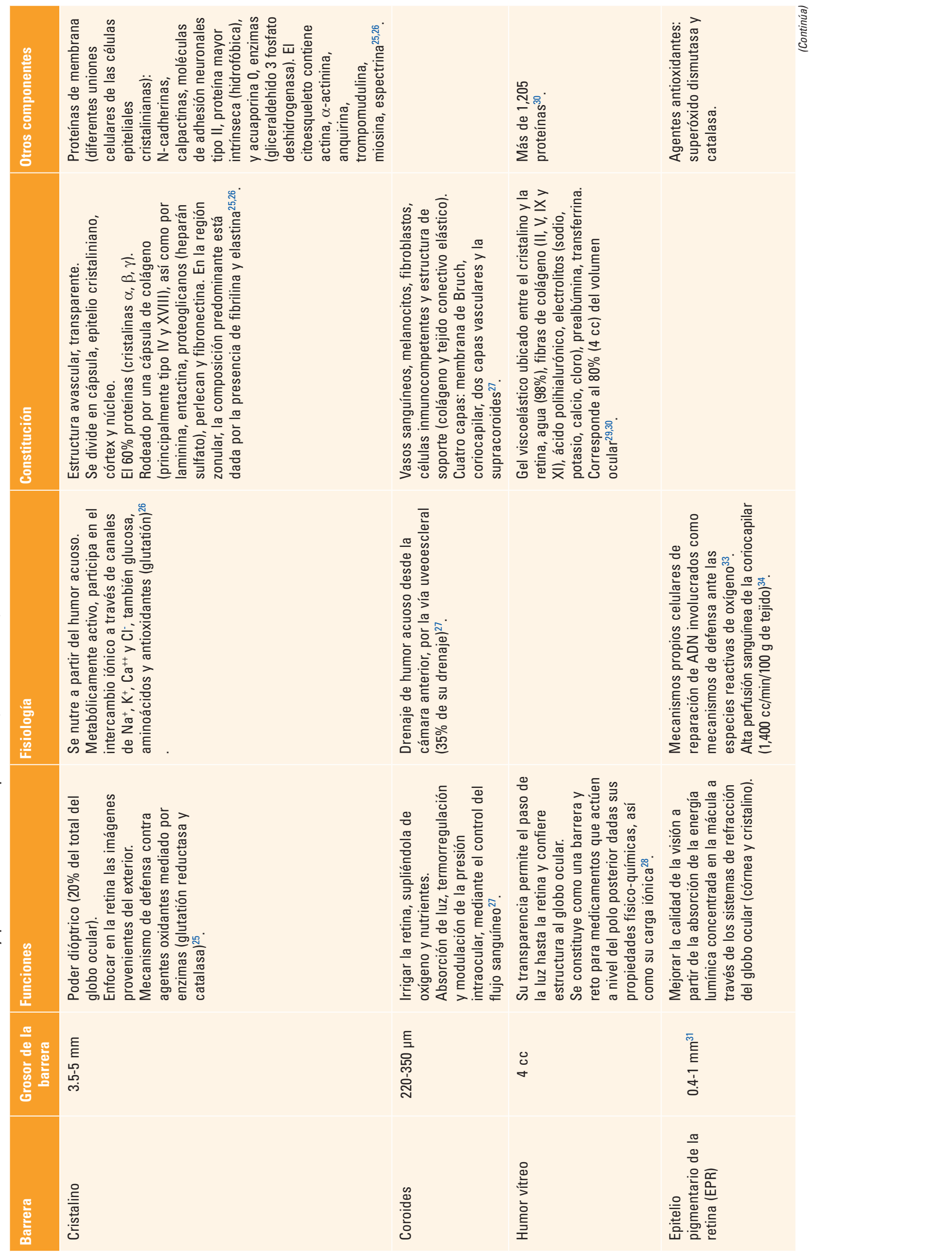



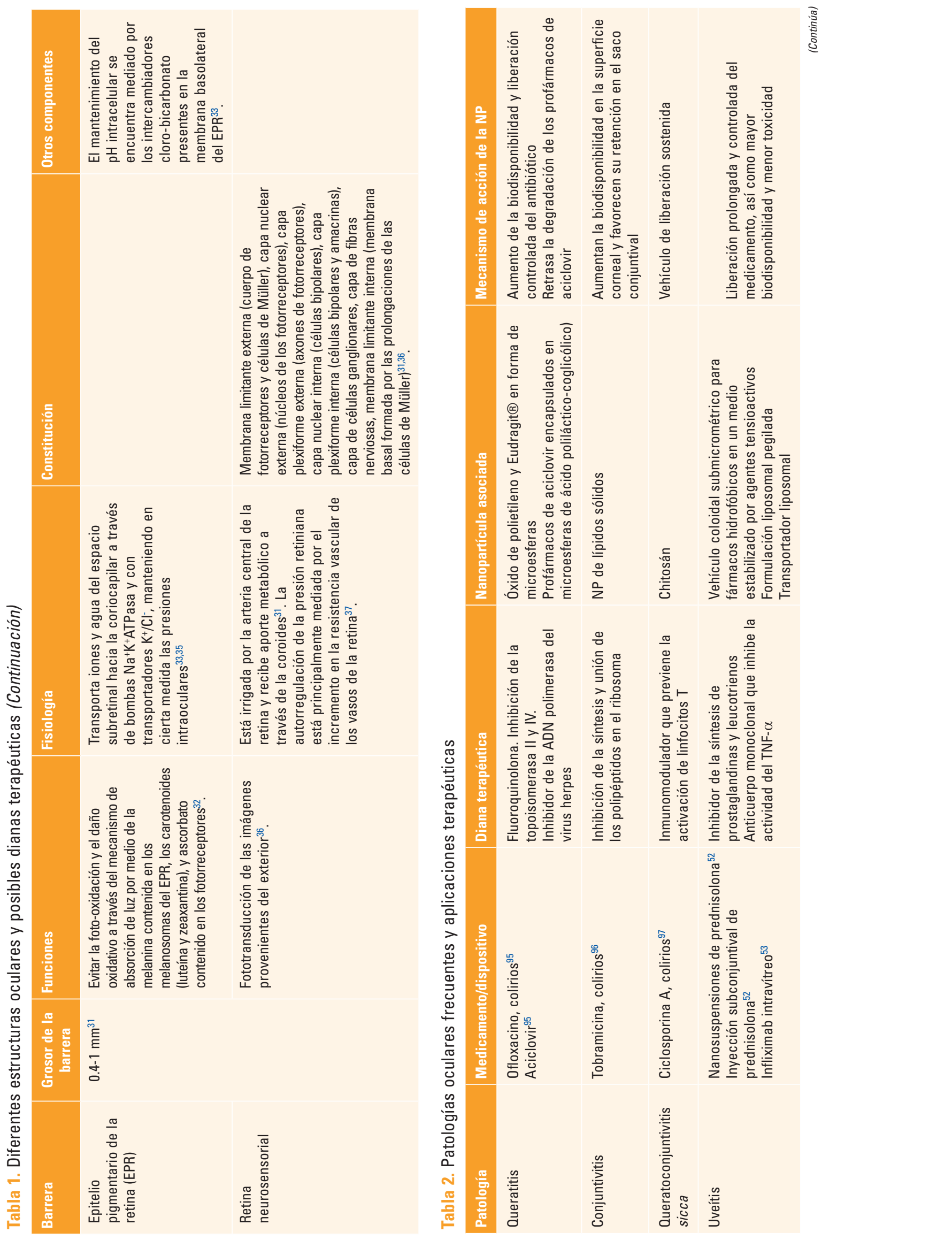

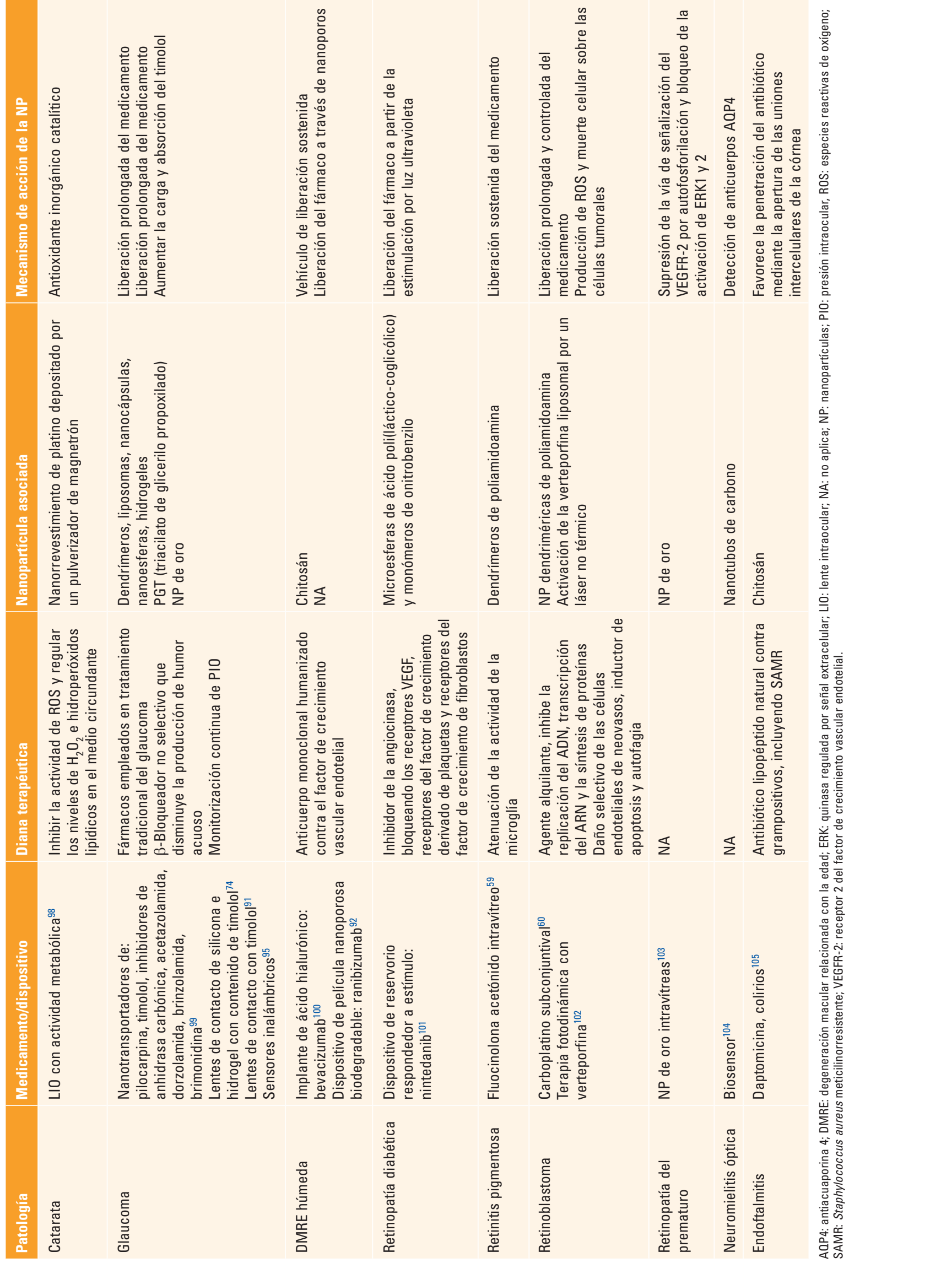


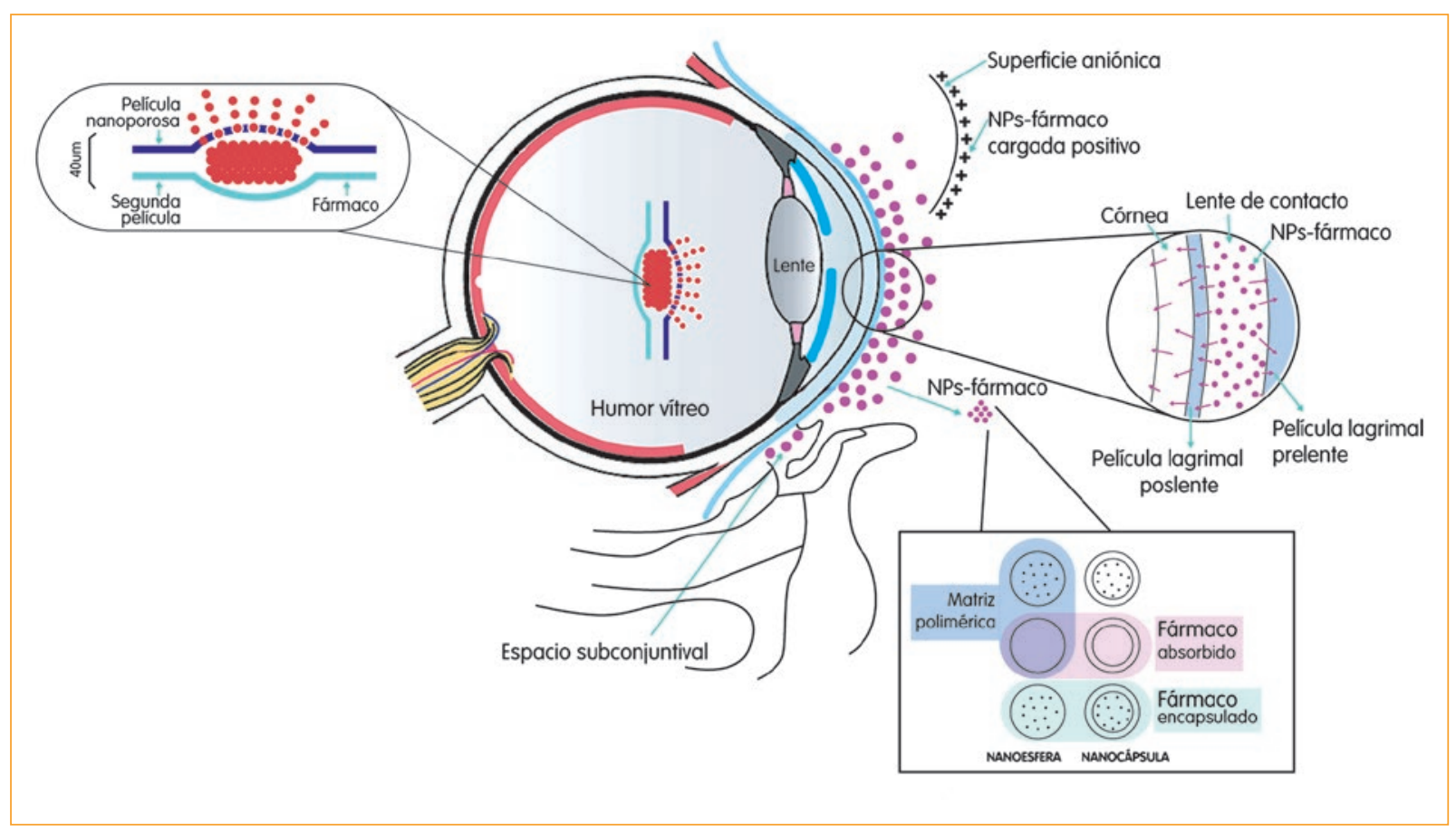

Figura 1. Administración de fármacos oftálmicos por medio de nanotecnología a través de las diferentes estructuras anatómicas oculares.

NP: nanopartículas.

\section{Conceptos de nanotecnología}

La nanotecnología es la disciplina centrada en el estudio, diseño, síntesis, manipulación y aplicación de materiales, aparatos y sistemas funcionales, mediante el control de la materia a nanoescala (1-100 nanómetros $)^{38}$.

Rápidamente se ha implementado el uso de la nanotecnología para el diagnóstico, tratamiento y control de diferentes enfermedades. Esta nueva rama científica se denomina nanomedicina. La evolución del conocimiento de la nanotecnología en áreas médico-quirúrgicas ha permitido el progreso de las aplicaciones en la oftalmología. Es así como se han diseñado novedosos nanosistemas oculares de diferentes formas y con características específicas para optimizar la biodisponibilidad de los medicamentos, al mejorar el tiempo de contacto y disminuir el proceso de eliminación del ojo ${ }^{39}$.

Existen muchos nanosistemas. Entre otros, los lentes de contacto cargados con nanopartículas para administrar acetazolamida en el tratamiento del glaucoma $^{40}$, los implantes subconjuntivales biodegradables para administrar ciclosporina A para el tratamiento del síndrome del ojo seco ${ }^{41,42}$, el desarrollo de sistemas de nanocoloidales basados en hidrogeles para la liberación ocular de diclofenaco ${ }^{43,44}$, los sistemas nanomicelares poliméricos para enfermedades inflamatorias ${ }^{45,46}$, y los transportadores de lípidos nanoestructurados para el suministro controlado de medicamentos oculares para infecciones ${ }^{47,48}$.

\section{Liposomas}

Los liposomas son vesículas de lípidos, con una o más bicapas de fosfolípidos, y encierran un núcleo acuoso. En función de su tamaño y el número de bicapas de fosfolípidos, los liposomas pueden clasificarse en vesículas unilamelares pequeñas (10 y $100 \mathrm{~nm}$ ), vesículas unilamelares grandes (100 y $300 \mathrm{~nm}$ ) y vesículas multilamelares, que contienen más de una sola bicapa de fosfolípido ${ }^{49}$. Los liposomas son ideales porque encapsulan fármacos tanto hidrofílicos como hidrofóbicos y demuestran una muy buena compatibilidad con los tejidos oculares ${ }^{50,51}$. Ejemplo del uso de este tipo de nanopartículas son las nanosuspensiones de prednisolona e infliximab intravítreo con nanotransportador liposomal ${ }^{52,53}$.

\section{Nanopartículas poliméricas}

Las micelas poliméricas son nanopartículas estructuradas en núcleo/cubierta formados por el autoensamblaje 
de copolímeros anfifílicos. La estructura núcleo/cubierta permite la encapsulación de fármacos hidrófobos en su núcleo hidrófobo. Debido a que el núcleo está protegido por la corona hidrófila, la biodisponibilidad del fármaco se prolonga significativamente al ser administrado por vía tópica en los tejidos oculares ${ }^{54,55}$. Un estudio experimental desarrollado por Mittal, et al. con timolol maleato tópico en conejos evidenció biocompatibilidad con la córnea y disminución de la PIO por tiempos más prolongados ${ }^{56}$.

\section{Nanosuspensiones}

Son dispersiones coloidales en las cuales los fármacos hidrófobos se dispersan uniformemente en un medio acuoso con la ayuda de surfactantes ${ }^{9}$. La prednisona, dexametasona, hidrocortisona y otros corticosteroides, por ejemplo, se han administrado a través de nanosuspensiones para el tratamiento de la inflamación del segmento anterior, sin los efectos secundarios esperados de aplicaciones de dosis grandes, como en el caso de catarata y neuropatía óptica glaucomatosa.

\section{Dendrímeros}

Los dendrímeros son macromoléculas monodispersas, con varios grupos finales reactivos que rodean una molécula pequeña y forman una cavidad interna. Su arquitectura ramificada en forma de árbol presenta una variedad de grupos terminales repetitivos. Especialmente los dendrímeros de baja generación pueden encapsular moléculas de fármacos hidrófobos en sus cavidades internas. Debido a esta estructura única, los dendrímeros permiten la solublilización de medicamentos poco solubles en agua. Además, los dendrímeros se pueden considerar como una imitación real de las proteínas globulares. Se les conoce como «proteínas artificiales», en función de sus propiedades sistemáticas, electroforéticas, de escala dimensional, y otras propiedades biomiméticas ${ }^{57,58}$.

Los medicamentos desarrollados con esta tecnología incluyen acetónido de fluocinolona intravítreo para el tratamiento de la retinitis pigmentosa, y el carboplatino subconjuntival para el manejo del retinoblastoma ${ }^{59,60}$.

\section{Nanomicelas}

Son sistemas de administración de fármacos compuestos de un núcleo hidrófobo y una cubierta hidrófila, los cuales permiten la disolución de fármacos hidrófobos, con la generación de una formulación acuosa transparente en la preparación para la administración de medicamentos en el segmento anterior del globo ocular. Uno de los medicamentos en los que se ha aplicado este tipo de nanotecnología es la ciclosporina. Un ensayo clínico de fase III demostró su efectividad, seguridad y rápida acción en el tratamiento de queratoconjuntivitis sicca ${ }^{61}$.

\section{Niosomas}

Los niosomas son vesículas tensioactivas no iónicas de dos capas, que pueden atrapar fármacos hidrófilos y lipófilos. Los niosomas son químicamente estables y su naturaleza no iónica hace que tengan una baja toxicidad $^{62}$. Gracias a su superficie hidrófila, los niosomas interactúan y atraviesan fácilmente la barrera de la película lagrimal, como resultado pueden alcanzar el tejido corneal/conjuntival ${ }^{63}$. Los niosomas se han evaluado como anticolinérgicos, antiglaucomatosos y antibióticos ${ }^{62,64,65}$. Las características más determinantes para su uso como vehículos para la administración de fármacos oculares son: tamaño de la vesícula, lo suficientemente grande como para resistir el drenaje mediante lagrimeo reflejo y el parpadeo; presentar formas con irregularidades que permitan encajar correctamente en el fondo de saco y alojarse en la superficie ocular; ser idealmente sensibles al calor para liberar el contenido del fármaco de manera controlada, pero a la vez, antes de removerse con el parpadeo y drenaje nasolagrimal ${ }^{66,67}$.

\section{Cubosomas}

La estructura del cubosoma consiste en una bicapa lipídica continua muy retorcida, con dos canales de agua congruentes que no se cruzan. En comparación con la estructura bicapa simple de los liposomas, los cubosomas tienen un área de superficie aumentada y una gran capacidad para encapsular diversas moléculas de sustancias hidrófilas, hidrófobas y anfifílicas. Los cubosomas poseen una estabilidad físico-química más alta que los liposomas, debido a la fuerte repulsión eléctrica y una gran proporción de bicapa lipídica ${ }^{68-70}$. El uso de dexametasona en colirios, asociada a este tipo de nanopartícula, evidencia una mayor disponibilidad del medicamento en el humor acuoso ${ }^{71}$.

\section{Hidrogeles}

Son una red de monómeros y retransmisores multifuncionales que reaccionan para formar una estructura flexible cargada de agua. Las redes de hidrogel se han 
estudiado exhaustivamente como un sistema de administración de fármacos controlado y sostenido, ya que la porosidad de su matriz se puede adaptar modificando la densidad del reticulado. Esta capacidad de cambio en función del entorno circundante tiene importantes implicaciones para los hidrogeles de formación in situ, aquellos que se reticulan cuando la temperatura aumenta desde la temperatura ambiente a la temperatura corporal, así como la liberación controlada de fármacos como resultado del $\mathrm{pH}$ o la fotoestimulación ${ }^{72,73}$. Los lentes de contacto de silicona e hidrogel con contenido de timolol de liberación controlada son una nueva tecnología desarrollada recientemente ${ }^{74}$, y resulta prometedora.

\section{Nanofibras poliméricas}

Las nanofibras son fibras sólidas de materiales que tienen diámetros por debajo del rango de micras, con una estructura porosa y un área de superficie muy alta.

Las nanofibras son estructuras fibrosas no tejidas, similares a la matriz extracelular, que consisten en un compuesto acuoso de fibrillas poliméricas altamente organizadas y proteoglicanos para apoyar la formación de tejido. Por esta razón, una de sus principales aplicaciones es la ingeniería de tejidos. Los dispositivos en los que se han desarrollado estudios son las queratoprótesis; esto, con el objetivo de salvaguardar la biocompatibilidad, fisiología y, por ende, la propiedad más importante de la córnea, su transparencia ${ }^{75,76}$.

Adicionalmente, gracias al diámetro tan pequeño y a un área de superficie excepcionalmente alta de las nanofibras, se puede cargar un contenido de fármaco significativamente mayor en una porción muy pequeña del parche ${ }^{77,78}$.

\section{Nanoformulaciones para el tratamiento de enfermedades oculares}

Gran parte de los productos oftálmicos del mercado son formulaciones tópicas para la administración de fármacos al segmento anterior. La mayor desventaja es que solo el $5 \%$ de la dosis instilada alcanza la cámara anterior, pero la que penetra en el segmento posterior es más pequeña, debido a las múltiples y complejas barreras anatómicas del globo ocular. La administración de fármacos oftálmicos de tamaño nano (Fig. 1) representa ventajas por su mayor solubilidad, mayor superficie disponible para disolución, mayor velocidad de disolución, mayor bioadhesión y penetración corneal. Se ha recomendado que las partículas midan menos de $10 \mu \mathrm{m}$ para minimizar la irritación de las estructuras oculares, disminuir el lagrimeo y el drenaje de la dosis instilada y, por lo tanto, aumentar la eficacia de un tratamiento ocular.

\section{Nanosuspensiones}

Las nanosuspensiones son dispersiones coloidales submicrométricas de partículas de fármacos puros en una fase líquida externa. Una característica importante de la nanosuspensión es el aumento de la solubilidad de saturación y, en consecuencia, un aumento en la velocidad de disolución del compuesto. En este sistema, los fármacos se cargan mediante la unión a la matriz o disueltos dentro, encapsulados 0 atrapados dentro de la estructura, y se genera un sistema versátil de suministro de fármacos, entre los cuales se incluyen las microemulsiones, liposomas, niosomas, dendrímeros y ciclodextrinas ${ }^{79,80}$.

Las ventajas del uso de nanopartículas incluyen el paso tópico mejorado de moléculas grandes y poco solubles en agua, como es el caso de los glucocorticoides o la ciclosporina para tratar las enfermedades inmunológicas que afectan la visión ${ }^{81}$. Otras moléculas grandes e inestables, como los ácidos nucleicos, administradas mediante nanopartículas, ofrecen resultados prometedores para la terapia de transferencia génica en el tratamiento de retinopatías ${ }^{82,83}$. La administración de medicamentos mediada por nanopartículas aumenta el tiempo de contacto del medicamento administrado con su tejido objetivo, como ocurre con la brimonidina, uno de los tratamientos tradicionales para el glaucoma, - los corticosteroides utilizados para tratar la uveítis autoinmune ${ }^{78,84}$. Algunas nanoformulaciones han permitido que el medicamento antiinflamatorio no esteroideo, indometacina, llegue a las estructuras internas posteriores del ojo utilizando la ruta transmucosa ${ }^{85}$. Entre las nuevas aplicaciones se mencionan el uso de las nanopartículas de oro que permiten la posibilidad de entrega dirigida para alcanzar tipos específicos de cáncer, como el melanoma coroideo, dejando intactas las células normales ${ }^{86}$.

\section{Lentes de contacto}

Los lentes de contacto son dispositivos poliméricos duros o blandos diseñados para adaptarse directamente a la córnea para corregir anomalías de refracción. Wichterle, et al., en 1965, patentaron la idea de usar lentes de contacto de hidrogel como dispositivos de suministro de medicamentos. En la patente se 
menciona la inclusión de medicamentos en la hidratación de lentes para ofrecer una mayor disponibilidad del fármaco durante su uso ${ }^{87}$.

La inclusión de nanopartículas cargadas de medicamentos dentro de la matriz polimérica del lente de contacto es una estrategia efectiva para la administración prolongada de fármacos. La incorporación del medicamento se logra con métodos como la impresión, el remojo simple y las nanopartículas coloidales ${ }^{88-90}$. El diámetro de la nanopartícula debe ser extremadamente pequeño para su uso en lentes de contacto para evitar que las partículas obstaculicen la visión del usuario. Por lo tanto, es necesario retrasar la liberación del medicamento a través de otros medios. Esto se puede lograr uniendo el fármaco con la partícula a través de un enlace químico escindible o diseñando una partícula de modo que el fármaco tenga una mayor afinidad por la partícula que por el material del lente circundante $^{89}$. Este enfoque permite la liberación sostenida, que puede ajustarse a las necesidades del paciente, desde unas pocas horas hasta varias semanas, y permite el tratamiento de patologías del segmento anterior.

Se han patentado diferentes nanopartículas, liderando los liposomas y microemulsiones que contienen el producto farmacéutico y luego son cargadas en los lentes de contacto. Los liposomas se han utilizado en varias aplicaciones de administración de fármacos debido a su alta biocompatibilidad, los lentes permanecen transparentes y liberan el medicamento durante unos días, con una liberación inicial que se atribuye al medicamento no encapsulado presente en el lente. Los lentes de contacto cargados con microemulsiones liberarán el fármaco durante 4 a 8 días, con un pico inicial que se le atribuya al fármaco sin encapsular ${ }^{89}$.

Jung, et al., en 2013, dispersaron las nanopartículas de timolol de PGT (triacrilato de glicerilo propoxilado) en lentes de contacto de silicona para administrar el medicamento durante 30 días. Los estudios preliminares en perros Beagle mostraron resultados prometedores para el glaucoma. La incorporación de nanopartículas en los hidrogeles de silicona provocó la reducción en las permeabilidades de iones y oxígeno, y un aumento en el módulo, y el impacto en cada una de estas propiedades era proporcional a la carga de partículas ${ }^{74}$. Maulvi, et al., en 2018, incorporaron nanopartículas de oro en los lentes de contacto y mejoraron la absorción de timolol de la solución disolvente del fármaco junto con resultados cinéticos y dinámicos satisfactorios in vivo, sin alterar las propiedades del lente ${ }^{91}$. Los dispositivos estudiados han presentado excelentes propiedades mecánicas, y los investigadores proponen que el material es adecuado para el suministro de medicamentos a partir de lentes de contacto reutilizables de uso diario.

\section{Implantes intraoculares}

Los implantes oculares son los nuevos tratamientos que buscan la liberación controlada de fármacos, al trabajar dosificaciones y cargas mayores de fármaco. Además, se presenta menor efecto secundario sistémico y mayor proximidad al sitio blanco, el segmento posterior del ojo. Se pueden emplear polímeros biodegradables 0 no biodegradables en los sistemas implantables oculares. Si bien los implantes biodegradables no necesitan ser removidos después de la inserción en el ojo, los no biodegradables requieren una intervención adicional para remover o rellenar los implantes, lo que conlleva costos adicionales y riesgos quirúrgicos intra o postoperatorios. Los desarrollos más recientes en sistemas implantables biodegradables son el implante ENV705 ${ }^{\mathrm{TM}}$ de Envisia Therapeutics y el dispositivo de película nanoporosa de Zordera ${ }^{92}$.

El dispositivo de película nanoporosa de Zordera consiste en una pastilla de medicamento intercalada entre dos capas delgadas de membrana biodegradable impermeable. Un lado tiene nanoporos del mismo diámetro que el activo, lo que permite que solo una molécula de fármaco salga del reservorio de cada poro a la vez. El dispositivo se inyecta en el vítreo; es muy delgado, con un diámetro de solo $40 \mu \mathrm{m}$ y una liberación sostenida de fármaco de orden casi 0 . Las capas de polímero se degradan en un momento posterior cuando se libera la mayor parte del medicamento, lo que elimina la necesidad de retirar el dispositivo. Este implante ofrece control sobre la velocidad de liberación al ajustar el tamaño de poro, y ha demostrado que administra ranibizumab durante cuatro meses de manera sostenida. Por lo tanto, puede convertirse en el mejor implante biodegradable para tratar afecciones retinianas crónicas ${ }^{93}$. Caso similar ocurre con la liberación de sirolimus en el polo posterior a través de este mismo dispositivo ${ }^{94}$.

\section{Aplicaciones clínicas}

Teniendo claridad de la anatomía, histología y fisiología de las diferentes barreras oculares, así como de las principales nanopartículas que se han desarrollado en el ámbito de la investigación, en la tabla 2 se mencionan las patologías oculares más frecuentes y con mayor impacto negativo en la agudeza visual en las 
que se ha involucrado la nanotecnología para su manejo en los diferentes sitios de acción de los tejidos del globo ocular (Fig. 1).

\section{Perspectivas}

Es amplia la gama de aplicaciones de la nanotecnología y la nanomedicina en el ámbito de la oftalmología. De diversas maneras, el uso de estos dispositivos y nanoformulaciones favorece la biodisponibilidad de los medicamentos, permite la difusión a través de las barreras anatómicas y podría disminuir los efectos adversos adjudicados al uso de los medicamentos oculares tópicos de manera convencional, y muy posiblemente disminuya, en alguna medida, intervenciones invasivas en el polo posterior, así como complicaciones secundarias al uso de ciertos medicamentos que requieran procedimientos quirúrgicos para su utilización. En últimas se optimizan los beneficios de los medicamentos y se disminuyen los impactos negativos, y se abre una gran ventana dentro del espectro de la llamada medicina personalizada, que muy probablemente requerirá nuevos estudios con diseños particulares para poblaciones con características individuales, así como el constante estudio en diversos modelos animales y de laboratorio.

\section{Conflicto de intereres}

Los autores declaran no tener ningún conflicto de intereses.

\section{Responsabilidades éticas}

Protección de personas y animales. Los autores declaran que para esta investigación no se han realizado experimentos en seres humanos ni en animales.

Confidencialidad de los datos. Los autores declaran que en este artículo no aparecen datos de pacientes.

Derecho a la privacidad y consentimiento informado. Los autores declaran que en este artículo no aparecen datos de pacientes.

\section{Bibliografía}

1. Salud OOMdl. Ceguera y discapacidad visual. 2019. Disponible en: https://www.who.int/es/news-room/fact-sheets/detail/blindness-and-visual-impairment.

2. Meng T, Kulkarni V, Simmers R, Brar V, Xu Q. Therapeutic implications of nanomedicine for ocular drug delivery. Drug discovery today. England: 2019 Elsevier Ltd; 2019.

3. NSTC. NATIONAL NANOTECHNOLOGY INITIATIVE - LEADING TO THE NEXT INDUSTRIAL REVOLUTION. Microscale Thermophysical Engineering. 2000;4(3):205-12.

4. Guchet X. What's in a word? The person of personalized (nano)medicine. Nanomedicine (Lond). 2015;10(20):3167-79.
5. Feng $Y$, Zhang $Y$, Ying C, Wang D, Du C. Corrigendum to 'Nanopore-based Fourth-generation DNA Sequencing Technology' [GPB 144 (2015) - GPB 13/1 (4-16)]. Genomics Proteomics Boinformatics. 2015;13(6):383.

6. Peck RW. Precision Medicine Is Not Just Genomics: The Right Dose for Every Patient. Ann Rev Pharmacol Toxicol. 2018;58:105-22.

7. Ventola CL. Progress in Nanomedicine: Approved and Investigational Nanodrugs. P \& T: a peer-reviewed journal for formulary management. 2017;42(12):742-55

8. Xu Q, Kambhampati SP, Kannan RM. Nanotechnology approaches for ocular drug delivery. Middle East Afr J Ophthalmol. India2013. p. 26-37.

9. Kamaleddin MA. Nano-ophthalmology: Applications and considerations. Nanomedicine. United States: 2017 Elsevier Inc; 2017. p. 1459-72.

10. Zarbin MA, Arlow T, Ritch R, Wang YY, Lai SK, Ensign LM, et al. Regenerative nanomedicine for vision restoration The microstructure and bulk rheology of human cervicovaginal mucus are remarkably resistant to changes in pH. Mayo Clin Proc. England United States: 2013 Mayo Foundation for Medical Education and Research. Published by Elsevier Inc; 2013. p. $1480-90$

11. Dartt DA, Willcox MD. Complexity of the tear film: importance in homeostasis and dysfunction during disease. Exp Eye Res. 2013;117:1-3.

12. Gulati S, Jain S. Ocular Pharmacology of Tear Film, Dry Eye, and Allergic Conjunctivitis. Handbook Exp Pharmacol. 2017;242:97-118.

13. Willcox MDP, Argueso P, Georgiev GA, Holopainen JM, Laurie GW, Millar TJ, et al. TFOS DEWS II Tear Film Report. Ocul Surf. 2017;15(3):366-403.

14. Sridhar MS. Anatomy of cornea and ocular surface. Indian J Ophthalmol. 2018;66(2):190-4.

15. Ma J, Wang Y, Wei $P$, Jhanji V. Biomechanics and structure of the cornea: implications and association with corneal disorders. Surv Ophthalmol. 2018;63(6):851-61.

16. Williams K, Watsky M. Gap junctional communication in the human corneal endothelium and epithelium. Curr Eye Res. 2002;25(1):29-36.

17. Kling S, Hafezi F. Corneal biomechanics - a review. Ophthalmic Physiol Opt. 2017;37(3):240-52.

18. Zavala J, Lopez Jaime GR, Rodriguez Barrientos CA, Valdez-Garcia J. Corneal endothelium: developmental strategies for regeneration. Eye (London, England). 2013;27(5):579-88.

19. Takahashi Y, Watanabe A, Matsuda H, Nakamura Y, Nakano T, Asamoto $\mathrm{K}$, et al. Anatomy of secretory glands in the eyelid and conjunctiva: a photographic review. Ophthal Plastic Rec Surg. 2013;29(3):215-9.

20. Gipson IK. Goblet cells of the conjunctiva: A review of recent findings. Progress Retinal Eye Res. 2016;54:49-63.

21. Galicia-Carreon J, Santacruz C, Hong E, Jimenez-Martinez MC. The ocular surface: from physiology to the ocular allergic diseases. Revista Alergia Mexico (Tecamachalco, Puebla, Mexico : 1993). 2013;60(4):172-83.

22. Watson PG, Young RD. Scleral structure, organisation and disease. A review. Exp Eye Res. 2004;78(3):609-23.

23. Davis-Silberman N, Ashery-Padan R. Iris development in vertebrates; genetic and molecular considerations. Brain Res. 2008;1192:17-28.

24. Civan MM, Macknight AD. The ins and outs of aqueous humour secretion. Exp Eye Res. 2004;78(3):625-31.

25. Aliancy JF, Mamalis N. Crystalline Lens and Cataract. In: Kolb H, Fernandez E, Nelson R, editors. Webvision: The Organization of the Retina and Visual System. Salt Lake City UT: Webvision; 2017

26. Assia El, Apple DJ. Side-view analysis of the lens. I. The crystalline lens and the evacuated bag. Arch Ophthalmol. (Chicago, III : 1960). 1992;110(1):89-93.

27. Nickla DL, Wallman J. The multifunctional choroid. Prog Retin Eye Res. 2010;29(2):144-68

28. Mains J, Wilson CG. The vitreous humor as a barrier to nanoparticle distribution. J Ocul Pharmacol Ther. 2013;29(2):143-50.

29. Mulla A, Massey KL, Kalra J. Vitreous humor biochemical constituents: evaluation of between-eye differences. Am J Forensic Med Pathol. 2005;26(2):146-9.

30. Murthy KR, Goel R, Subbannayya Y, Jacob HK, Murthy PR, Manda SS, et al. Proteomic analysis of human vitreous humor. Clin Proteomics. 2014;11(1):29.

31. Willoughby CE, Ponzin D, Ferrari S, Lobo A, Landau K, Omidi Y. Anatomy and physiology of the human eye: effects of mucopolysaccharidoses disease on structure and function - a review. Clin Exp Ophthal. 2010;38(s1):2-11.

32. Simo R, Villarroel M, Corraliza L, Hernandez C, Garcia-Ramirez M. The retinal pigment epithelium: something more than a constituent of the blood-retinal barrier--implications for the pathogenesis of diabetic retinopathy. J Biomed Biotechnol. 2010;2010:190724.

33. Strauss $O$. The retinal pigment epithelium in visual function. Physiological Rev. 2005;85(3):845-81.

34. Sparrow JR, Hicks D, Hamel CP. The retinal pigment epithelium in health and disease. Curr Mol Med. 2010;10(9):802-23.

35. Boulton M, Dayhaw-Barker P. The role of the retinal pigment epithelium: topographical variation and ageing changes. Eye (London, England). 2001;15(Pt 3):384-9.

36. Gupta MP, Herzlich AA, Sauer T, Chan CC. Retinal Anatomy and Pathology. Develop Ophthalmol. 2016;55:7-17. 
37. Harris A, Ciulla TA, Chung HS, Martin B. Regulation of retinal and optic nerve blood flow. Arch Ophthalmol (Chicago, III : 1960). 1998;116(11):1491-5.

38. Gómez M. Nanomateriales, Nanopartículas y Síntesis verde. Repert Med Cir. 2018;27(2):75-80

39. Weng Y, Liu J, Jin S, Guo W, Liang X, Hu Z. Nanotechnology-based strategies for treatment of ocular disease. Acta Pharm Sin B. 2017;7(3):281-91.

40. Prakash M, Dhesingh RS. Nanoparticle Modified Drug Loaded Biodegradable Polymeric Contact Lenses for Sustainable Ocular Drug Delivery. Current drug delivery. United Arab Emirates: Bentham Science Publishers; For any queries, please email at epub@benthamscience.org.; 2017. p. 555-65.

41. Yavuz B, Bozdag Pehlivan S, Kaffashi A, Calamak S, Ulubayram K Palaska $E$, et al. In vivo tissue distribution and efficacy studies for cyclosporin A loaded nano-decorated subconjunctival implants Comparative study of human embryonic stem cells (hESC) and human induced pluripotent stem cells (hiPSC) as a treatment for retinal dystrophies. Drug delivery. England United States 2016. p. 3279-84

42. Pehlivan SB, Yavuz B, Calamak S, Ulubayram K, Kaffashi A, Vural I, et al. Preparation and in vitro/in vivo evaluation of cyclosporin A-loaded nanodecorated ocular implants for subconjunctival application. J Pharm Sci. 2015;104(5):1709-20.

43. Li X, Zhang Z, Chen $\mathrm{H}$. Development and evaluation of fast forming nano-composite hydrogel for ocular delivery of diclofenac. Int J Pharm. 2013;448(1):96-100

44. Li X, Zhang Z, Li J, Sun S, Weng Y, Chen H. Diclofenac/biodegradable polymer micelles for ocular applications. Nanoscale. 2012;4(15):4667-73.

45. Salama $A H$, Shamma RN. Tri/tetra-block co-polymeric nanocarriers as a potential ocular delivery system of lornoxicam: in-vitro characterization, and in-vivo estimation of corneal permeation. International journal of pharmaceutics. 2015;492(1-2):28-39.

46. Silva-Abreu M, Calpena AC, Espina M, Silva AM, Gimeno A, Egea MA et al. Optimization, Biopharmaceutical Profile and Therapeutic Efficacy of Pioglitazone-loaded PLGA-PEG Nanospheres as a Novel Strategy for Ocular Inflammatory Disorders. Pharm Res. 2018;35(1):11.

47. Ustundag-Okur N, Gokce EH, Bozbiyik DI, Egrilmez S, Ertan G, Ozer O. Novel nanostructured lipid carrier-based inserts for controlled ocular drug delivery: evaluation of corneal bioavailability and treatment efficacy in bacterial keratitis. Exp Op Drug Deliv. 2015;12(11):1791-807.

48. Ustundag-Okur N, Gokce EH, Bozbiyik DI, Egrilmez S, Ozer O, Ertan G. Preparation and in vitro-in vivo evaluation of ofloxacin loaded ophthalmic nano structured lipid carriers modified with chitosan oligosaccharide lactate for the treatment of bacterial keratitis. Eur J Pharm Sci. 2014;63:204-15

49. Kaiser JM, Imai H, Haakenson JK, Brucklacher RM, Fox TE, Shanmugavelandy SS, et al. Nanoliposomal minocycline for ocular drug delivery. Nanomedicine. 2013;9(1):130-40

50. Campos EJ, Campos A, Martins J, Ambrosio AF, Bisht R, Mandal A, et al Opening eyes to nanomedicine: Where we are, challenges and expectations on nanotherapy for diabetic retinopathy Nanocarrier mediated retinal drug delivery: overcoming ocular barriers to treat posterior eye diseases Intraocular application of gold nanodisks optically tuned for optical coherence tomography: inhibitory effect on retinal neovascularization without unbearable toxicity Surface plasmon-enhanced fluorescence on Au nanohole array for prostate-specific antigen detection. Nanomedicine. United States New Zealand: 2017 Elsevier Inc 2017 Wiley Periodicals, Inc.; 2017. p. $2101-13$.

51. Honda M, Asai T, Oku N, Araki Y, Tanka M, Ebihara N. Liposomes and nanotechnology in drug development: focus on ocular targets. Int $\mathrm{J} \mathrm{Na}$ nomedicine. New Zealand; 2013. p. 495-503.

52. Wong CW, Czarny B, Metselaar JM, Ho C, Ng SR, Barathi AV, et al Evaluation of subconjunctival liposomal steroids for the treatment of experimental uveitis. Scientific Reports. 2018;8(1):6604.

53. Zhang R, Qian J, Li X, Yuan Y. Treatment of experimental autoimmune uveoretinitis with intravitreal injection of infliximab encapsulated in liposomes. Br J Ophthal. 2017;101(12):1731-8.

54. Tsai CH, Wang PY, Lin IC, Huang H, Liu GS, Tseng CL. Ocular Drug Delivery: Role of Degradable Polymeric Nanocarriers for Ophthalmic Application. Inter J Molec Sci. 2018;19(9).

55. Alhalafi AM. Applications of polymers in intraocular drug delivery systems. Oman J Ophthalmol. 2017;10(1):3-8

56. Mittal N, Kaur G. Investigations on Polymeric Nanoparticles for Ocular Delivery. Adv Polymer Tech. 2019;1316249.

57. Yavuz B, Pehlivan SB, Unlu N. Dendrimeric systems and their applications in ocular drug delivery. Sci World J. 2013;2013:732340.

58. Kambhampati SP, Kannan RM, Zarbin MA, Montemagno C, Leary JF, Ritch $\mathrm{R}$, et al. Dendrimer nanoparticles for ocular drug delivery Nanomedicine for the treatment of retinal and optic nerve diseases Low molecular weight oligochitosans for non-viral retinal gene therapy Downregulation of VEGF mRNA expression by triamcinolone acetonide acetate-loaded chitosan derivative nanoparticles in human retinal pigment epithelial cells. J Ocul Pharmacol Ther. 2013;29(2):151-65.
59. Iezzi R, Guru BR, Glybina IV, Mishra MK, Kennedy A, Kannan RM. Dendrimer-based targeted intravitreal therapy for sustained attenuation of neuroinflammation in retinal degeneration. Biomaterials. 2012;33(3):979-88.

60. Kang SJ, Durairaj C, Kompella UB, O'Brien JM, Grossniklaus HE. Subconjunctival nanoparticle carboplatin in the treatment of murine retinoblastoma. Arch Ophthalmol (Chicago, III : 1960). 2009;127(8):1043-7.

61. Mandal A, Gote V, Pal D, Ogundele A, Mitra AK. Ocular Pharmacokinetics of a Topical Ophthalmic Nanomicellar Solution of Cyclosporine (Cequa(R)) for Dry Eye Disease. Pharma Res. 2019;36(2):36.

62. Perini G, Saettone MF, Carafa M, Santucci E, Alhaique F. Niosomes as carriers for ophthalmic drugs: in vitro/in vivo evaluation. Bollettino Chimico farmaceutico. 1996;135(2):145-6..

63. Abdelkader H, Alani AW, Alany RG. Recent advances in non-ionic surfactant vesicles (niosomes): self-assembly, fabrication, characterization, drug delivery applications and limitations. Drug Delivery. 2014;21(2):87-100.

64. Yadav KS, Rajpurohit R, Sharma S. Glaucoma: Current treatment and impact of advanced drug delivery systems. Life Sciences. 2019;221:362-76.

65. Khalil RM, Abdelbary GA, Basha M, Awad GE, El-Hashemy HA Design and evaluation of proniosomes as a carrier for ocular delivery of lomefloxacin HCl. J Lipo Res. 2017;27(2):118-29.

66. Battaglia L, Serpe L, Foglietta F, Muntoi E, Gallarate M, Del Pozo Rodriguez A, et al. Application of lipid nanoparticles to ocular drug delivery. Ex Opin Drug Delivery. 2016;13(12):1743-57.

67. Gan L, Wang J, Jiang M, Bartlett H, Ouyang D, Eperjesi F, et al. Recent advances in topical ophthalmic drug delivery with lipid-based nanocarriers. Drug Discov Today. 2013;18(5-6):290-7.

68. Han S, Shen JQ, Gan Y, Geng HM, Zhang XX, Zhu CL, et al. Novel vehicle based on cubosomes for ophthalmic delivery of flurbiprofen with ow irritancy and high bioavailability. Acta Pharmacologica Sinica. 2010;31(8):990-8.

69. Huang J, Peng T, Li Y, Zhan Z, Zeng Y, Huang Y, et al. Ocular Cubosome Drug Delivery System for Timolol Maleate: Preparation, Characterization, Cytotoxicity, Ex Vivo, and In Vivo Evaluation. AAPS PharmSciTech. 2017;18(8):2919-26.

70. Hartnett TE, O'Connor AJ, Ladewig K. Cubosomes and other potentia ocular drug delivery vehicles for macromolecular therapeutics. Ex Opin Drug Delivery. 2015;12(9):1513-26.

71. Gan L, Han S, Shen J, Zhu J, Zhu C, Zhang X, et al. Self-assembled liquid crystalline nanoparticles as a novel ophthalmic delivery system for dexamethasone: Improving preocular retention and ocular bioavailability. Int J Pharm. 2010;396(1-2):179-87.

72. Cooper RC, Yang $\mathrm{H}$. Hydrogel-based ocular drug delivery systems: Emerging fabrication strategies, applications, and bench-to-bedside manufacturing considerations. J Control Release. 2019;306:29-39.

73. Kirchhof S, Goepferich AM, Brandl FP. Hydrogels in ophthalmic applications. Eur J Pharm Biopharm. 2015;95(Pt B):227-38.

74. Jung HJ, Abou-Jaoude M, Carbia BE, Plummer C, Chauhan A. Glaucoma therapy by extended release of timolol from nanoparticle loaded silicone-hydrogel contact lenses. J Control Release. 2013;165(1):82-9.

75. Wu Z, Kong B, Liu R, Sun W, Mi S. Engineering of Corneal Tissue through an Aligned PVA/Collagen Composite Nanofibrous Electrospun Scaffold. Nanomaterials (Basel, Switzerland). 2018;8(2).

76. Myung D, Duhamel PE, Cochran JR, Noolandi J, Ta CN, Frank CW. Development of hydrogel-based keratoprostheses: a materials perspective. Biotech Progress. 2008;24(3):735-41.

77. Zhan J, Singh A, Zhang Z, Huang L, Elisseeff JH. Multifunctional aliphatic polyester nanofibers for tissue engineering. Biomatter. 2012;2(4):202-12.

78. Gagandeep, Garg T, Malik B, Rath G, Goyal AK. Development and characterization of nano-fiber patch for the treatment of glaucoma. Eu $\mathrm{J}$ Pharm Sci. 2014:53:10-6.

79. Nagarwal RC, Kant S, Singh PN, Maiti P, Pandit JK. Polymeric nanoparticulate system: a potential approach for ocular drug delivery. J Control Release. 2009;136(1):2-13

80. Morrison PW, Khutoryanskiy VV. Advances in ophthalmic drug delivery. Ther Delivery. 2014;5(12):1297-315

81. Diebold $Y$, Calonge M. Applications of nanoparticles in ophthalmology. Progress Retin Eye Res. 2010;29(6):596-609.

82. Park K, Chen Y, Hu Y, Mayo AS, Kompella UB, Longeras R, et al. Nanoparticle-mediated expression of an angiogenic inhibitor ameliorates ischemia-induced retinal neovascularization and diabetes-induced retinal vascular leakage. Diabetes. 2009;58(8):1902-13.

83. del Pozo-Rodriguez A, Delgado D, Gascon AR, Solinis MA. Lipid nanoparticles as drug/gene delivery systems to the retina. J Ocul Pharmacol Ther. 2013;29(2):173-88.

84. Lalu L, Tambe V, Pradhan D, Nayak K, Bagchi S, Maheshwari R, et al. Novel nanosystems for the treatment of ocular inflammation: Current paradigms and future research directions. J Control Release. Netherlands: 2017. Published by Elsevier B.V.; 2017. p. 19-39.

85. Balguri SP, Adelli GR, Majumdar S. Topical ophthalmic lipid nanoparticle formulations (SLN, NLC) of indomethacin for delivery to the posterior segment ocular tissues. European journal of pharmaceutics and biopharmaceutics : official journal of Arbeitsgemeinschaft fur Pharmazeutische Verfahrenstechnik eV. 2016;109:224-35. 
86. Zabihzadeh M, Rezaee H, Hosseini SM, Feghhi M, Danyaei A, Hoseini-Ghahfarokhi M. Improvement of dose distribution in ocular brachytherapy with (125)I seeds 20-mm COMS plaque followed to loading of choroidal tumor by gold nanoparticles. J Cancer Res Ther. 2019;15(3):504-11.

87. Wichterle O, Lim D, inventors; Cross-linked hydrophilic polymers and articles made therefrom. US1965.

88. Dubald M, Bourgeois S, Andrieu V, Fessi H. Ophthalmic Drug Delivery Systems for Antibiotherapy-A Review. Pharmaceutics. 2018;10(1).

89. Dixon P, Shafor C, Gause S, Hsu KH, Powell KC, Chauhan A. Therapeutic contact lenses: a patent review. Ex Op Ther Patents. 2015;25(10):1117-29.

90. Guzman-Aranguez A, Fonseca B, Carracedo G, Martin-Gil A, Martinez-Aguila A, Pintor J. Dry Eye Treatment Based on Contact Lens Drug Delivery: A Review. Eye Contact Lens. 2016;42(5):280-8.

91. Maulvi FA, Patil RJ, Desai AR, Shukla MR, Vaidya RJ, Ranch KM, et al. Effect of gold nanoparticles on timolol uptake and its release kinetics from contact lenses: In vitro and in vivo evaluation. Acta Biomaterialia. 2019;86:350-62

92. Yasin MN, Svirskis D, Seyfoddin A, Rupenthal ID. Implants for drug delivery to the posterior segment of the eye: a focus on stimuli-responsive and tunable release systems. J Control Release. 2014;196:208-21.

93. Bernards D, Bhisitkul R, Deasi T. Zero-Order Sustained Drug Delivery to the Retina From a Nanoporous Film Device. On Drug Deliv Mag. 2014;48:20-1.

94. Lance KD, Good SD, Mendes TS, Ishikiriyama M, Chew P, Estes LS, et al. In Vitro and In Vivo Sustained Zero-Order Delivery of Rapamycin (Sirolimus) From a Biodegradable Intraocular Device. Invest Ophthalmo Visual Sci. 2015;56(12):7331-7.

95. Sharaf MG, Cetinel S, Heckler L, Damji K, Unsworth L, Montemagno C. Nanotechnology-Based Approaches for Ophthalmology Applications: Therapeutic and Diagnostic Strategies. Asia Pac J Ophthalmol (Phila). 2014;3(3):172-80.
96. Bachu RD, Chowdhury P, Al-Saedi ZHF, Karla PK, Boddu SHS. Ocular Drug Delivery Barriers-Role of Nanocarriers in the Treatment of Anterior Segment Ocular Diseases. Pharmaceutics. 2018;10(1).

97. De Campos AM, Sanchez A, Alonso MJ. Chitosan nanoparticles: a new vehicle for the improvement of the delivery of drugs to the ocular surface. Application to cyclosporin A. Int J Pharm. 2001;224(1-2):159-68.

98. Babizhayev MA. Coated with nanomaterials intraocular lenses, ophthalmic and human body implantable devices with high catalytic antioxidant activities: a new nanotechnology strategy of peroxidase cellular enzyme mimics increasing the biocompatibility and therapeutic deployment of the medical prosthetic device. Recent Pat Drug Deliv Formul. 2013;7(1):39-65.

99. Occhiutto ML, Maranhao RC, Costa VP, Konstas AG. Nanotechnology for Medical and Surgical Glaucoma Therapy-A Review. Adv Ther. 2020;37(1):155-99.

100. Badiee P, Varshochian R, Rafiee-Tehrani M, Abedin Dorkoosh F, Khoshayand MR, Dinarvand R. Ocular implant containing bevacizumab-loaded chitosan nanoparticles intended for choroidal neovascularization treatment. J Biomed Materials Res Part A. 2018;106(8):2261-71.

101. Huu VA, Luo J, Zhu J, Patel S, Boone A, Mahmoud E, et al. Light-responsive nanoparticle depot to control release of a small molecule angiogenesis inhibitor in the posterior segment of the eye. J Control Release. 2015;200:71-7.

102. Aleassa EM, Xing M, Keijzer R. Nanomedicine as an innovative therapeutic strategy for pediatric cancer. Ped Surg Inter. 2015;31(7):611-6.

103. Kim JH, Kim MH, Jo DH, Yu YS, Lee TG. The inhibition of retinal neovascularization by gold nanoparticles via suppression of VEGFR-2 activation. Biomaterials. 2011:32(7):1865-71.

104. Son M, Kim D, Park KS, Hong S, Park TH. Detection of aquaporin-4 antibody using aquaporin-4 extracellular loop-based carbon nanotube biosensor for the diagnosis of neuromyelitis optica. Biosensors ioelectronics. 2016;78:87-91.

105. Silva NC, Silva S, Sarmento B, Pintado M. Chitosan nanoparticles for daptomycin delivery in ocular treatment of bacterial endophthalmitis. Drug delivery. 2015;22(7):885-93. 